\title{
Estimativa do impacto das condições meteorológicas sobre a carga de trabalho dos controladores de tráfego aéreo na TMA-SP
}

\author{
Adilson Cleómenes Rocha ${ }^{1}$, Maria Paulete Pereira Martins ${ }^{2}$, Alexandre Luiz Dutra Bastos ${ }^{3 凶}$ \\ ${ }^{1}$ Instituto Nacional de Pesquisas Espaciais, acr_64@yahoo.com.br \\ ${ }^{2}$ Instituto Nacional de Pesquisas Espaciais, maria.paulete@cptec.inpe.br \\ 3Instituto de Controle do Espaço Aéreo, bastoscta@gmail.com
}

\section{Recebido:}

20 de agosto de 2016

Aceito para publicação:

27 de outubro de 2017

Publicado:

30 de dezembro de 2017

Editor de área:

Li Weigang

\section{Palavras-chaves:}

Carga de trabalho,

Gerenciamento de fluxo de tráfego

aéreo,

Condições de tempo adversas.

\section{Keywords:}

Workload

Air traffic management,

Adverse weather conditions.

DOI:10.14295/transportes.v25i4.1233

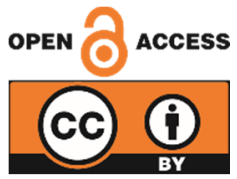

\begin{abstract}
RESUMO
Este estudo tem por objetivo estimar o impacto provocado por determinadas condições de tempo sobre a carga de trabalho dos ATCO que operam na TMA-SP. Para tal, foram simulados oito dias específicos no simulador TAAM, sendo sete deles com presença de mau tempo para a aviação. De modo geral, os resultados apontam para o aumento da carga laboral dos controladores, afetando setores importantes onde o movimento de voos já é considerado intenso. São identificadas condições e horários críticos que poderão servir de base para a realização de planejamentos estratégicos da segurança e eficiência operacional, no contexto do gerenciamento de tráfego aéreo. É importante que os órgãos responsáveis pelas ações de gerenciamento de fluxo de tráfego aéreo os considerem, pois isso poderá contribuir para a minimização dos impactos provocados pelos atrasos sobre custos diretos e indiretos das empresas aéreas, bem como sobre os usuários do transporte aéreo. Academicamente, percebe-se a necessidade da realização de pesquisas sobre a automatização de ações operacionais com a proposta de mitigar a sobrecarga de trabalho dos controladores.
\end{abstract}

\section{ABSTRACT}

This study aims to estimate the impact caused by certain weather conditions on the workload of the ATCO who operate in the TMA-SP. In order to do so, eight specific days were simulated in the TAAM simulator, seven of which contained different bad weather conditions for aviation. The results show an increase in the controllers' workload, which affects important sectors where the movement of flights is already considered heavy. Critical weather conditions and times are identified and can serve as the basis for strategic planning of safety and operational efficiency in the context of air traffic management. It is important that the one responsible for air traffic flow management actions consider them, as this can help minimize the impacts caused by delays on airlines' direct and indirect costs as well as on air transport users. Academically, it is necessary to carry out research on the automation of operational actions with the purpose of reducing the controllers' workload.
\end{abstract}

\section{INTRODUÇÃO}

Para Krozel et al. (2007), a capacidade de um espaço aéreo é definida como o número máximo de aeronaves por unidade de tempo que pode ser acomodado seguramente em um dado espaço aéreo controlado, considerando as restrições relativas ao piloto e à região voada. É um parâmetro fundamental para a determinação da condição operacional de um sistema ATC (Air Traffic Control). A capacidade de um espaço aéreo influencia e sofre influência direta do gerenciamento de fluxo do tráfego aéreo utilizado.

Condições meteorológicas adversas podem reduzir significativamente a capacidade do espaço aéreo em rota e/ou nas regiões das áreas terminais que envolvem aeroportos. Em função da saturação de capa- 
cidade, um aumento substancial no trabalho dos controladores pode gerar ou potencializar atrasos e congestionamentos no transporte aéreo, além de diminuir sua eficácia.

Este estudo estima a carga de trabalho dos controladores de tráfego aéreo que operam na Área de Controle Terminal de São Paulo (TMA-SP) em determinadas condições de tempo, seguindo uma metodologia específica. Isso foi feito por meio da realização de oito simulações de tráfego aéreo no Total Airspace and Airport Modeller (TAAM). Elas foram feitas para dias predeterminados do ano de 2014.

O primeiro dia utilizado na simulação foi aleatório, e, nesse caso, não foram consideradas as condições meteorológicas em qualquer região do Brasil. Ele serviu como referência de uma condição hipoteticamente ideal. Outros sete dias foram selecionados tomando como base os dias com maiores atrasos nos voos, a nível nacional. Nesses casos, identificaram-se as condições de tempo que estiveram presentes e que interferiram na evolução do tráfego aéreo civil. A base de dados dos voos considerados nesses dias foi obtida do Centro de Gerenciamento da Navegação Aérea (CGNA) e da Agência Nacional de Aviação Civil (ANAC).

Os resultados observados são de extrema relevância, pois, operacionalmente, poderão servir para auxiliar nos planejamentos relativos à segurança, regularidade e eficiência das operações aéreas. Com eles, os gestores operacionais das Células de Gerenciamento de Fluxo (Flow Management Cells - FMC), existentes nos Centros de Controle de Áreas (Area Control Center - ACC) impactados, bem como do Centro de Controle de Aproximação de São Paulo (APP-SP), poderão estabelecer novas estratégias de emprego de pessoal ATCO (Air Traffic Controller), de acordo com possíveis demandas horárias futuras decorrentes de condições meteorológicas adversas nos setores mais impactados. Além disso, também podem servir como fonte de informações numa possível reanálise da estrutura vertical e horizontal e da organização dos setores hoje estabelecidos, com o propósito de melhorar ainda mais a condição das rotas de chegadas e/ou partidas para aeroportos presentes na TMA-SP.

Academicamente, este estudo produz informações que poderão fomentar pesquisas e desenvolvimentos de algoritmos capazes de automatizar o ambiente ATM, diante de possíveis condições meteorológicas, além de fornecer dados que auxiliem na integração de sistemas de previsão do tempo aos de gerenciamento do tráfego aéreo.

O desenvolvimento de algoritmos capazes de automatizar as ações ATM contribuirá para a redução da subjetividade e dos efeitos das decisões operacionais ineficientes quando em presença de diversos tipos de mau tempo para a aviação. Caso seja adotado, também diminuirá o número de medidas ATM por parte do CGNA, das FMC e dos controladores nas posições operacionais, reduzindo suas respectivas cargas de trabalho.

A integração dos sistemas de meteorologia ao sistema de gerenciamento de tráfego aéreo pode contribuir com a identificação de rotas, níveis de voo, locais e horários onde as condições de tempo são mais impactantes na TMA-SP. Ela poderá, por exemplo, contribuir para a definição de técnicas e ações capazes de evitar as condições convectivas de tempo durante o voo, inclusive auxiliando no desenvolvimento de medidas automatizadas descritas no caso anterior.

\subsection{0 tráfego aéreo no brasil}

O ano de 2014 foi atípico para o cenário do transporte aéreo nacional. Nele se destaca a realização da Copa do Mundo da FIFA, as eleições presidenciais e a desaceleração das atividades econômicas. Mesmo nesse contexto, o transporte aéreo realizou o número recorde de 117 milhões de passageiros pagos, tendo sido 95,9 milhões em voos domésticos e 21,3 milhões em voos internacionais. 0 crescimento médio nesses dois mercados foi de 11,3\% e 7,9\% ao ano, respectivamente (ANAC, 2014). Quando se considera o movimento de decolagens domésticas, verifica-se que os três aeroportos da área de estudo deste trabalho (Aeroporto Internacional de Guarulhos - SBGR, Aeroporto Internacional de São Paulo - SBSP, e Aeroporto internacional de Campinas - SBKP) contribuem com mais de $25 \%$ do total, mesmo considerando um país de dimensão continental como o nosso. 
No espaço aéreo de responsabilidade do Brasil, todos os eventos relacionados ao voo são regulamentados pelo Departamento de Controle do Espaço Aéreo (DECEA), que promove ações diretas sobre o Sistema de Controle do Espaço Aéreo (SISCEAB). Nesse sentido, as atividades relativas ao ATM (Air Traffic Management) são regulamentadas por DECEA (2014a; 2014c; 2012; 2010). 0 ATC (Air Traffic Control) segue as normas definidas por DECEA (2014b, 2014c). A região de estudo deste trabalho possui ainda orientações específicas relacionadas aos órgãos de Controle de Aproximação (Approach Control $A P P$ ) de São Paulo e descritas em SRPV-SP (2014). Diversos procedimentos especiais também são regulamentados pelo DECEA por meio de Circulares de Informações Aeronáuticas (Aeronautical Information Circulars - AIC) e outras publicações afins.

\subsection{Carga de trabalho de controladores de tráfego aéreo}

Para o DECEA (2014a), a carga de trabalho do controlador de tráfego aéreo é o tempo utilizado pelo ATCO no processamento de todas as tarefas que uma posição de controle exige, em um intervalo de tempo, com o objetivo de manter o tráfego rápido, seguro e ordenado.

No contexto do estudo da sobrecarga de trabalho do controlador, Mogfort et al. (1995) fazem uma revisão bibliográfica sobre as operações em órgãos ATC, identificando os principais fatores relacionados à complexidade do espaço aéreo. Erzberger (2004) põe em destaque a importância da automatização de ações dos controladores com o objetivo de minimizar riscos e melhorar os benefícios sobre o espaço aéreo. Tabaruela et al. (2012) consideram indispensável estimar corretamente todos os fatores que afetam a capacidade do espaço aéreo, entre eles a carga de trabalho dos controladores. Já Barnhart et al. (2012) realizam sua pesquisa colocando luz nas iniciativas que as empresas aéreas e as autoridades de aviação civil devem adotar como medidas táticas de tráfego, em resposta às dinâmicas das condições reais encontradas, inclusive diante de condições de tempo significativas ou ruptura no planejamento de voo das empresas aéreas.

No Brasil, Baum e Müller (2008), seguindo Teixeira (2007), realizaram pesquisas sobre a carga de trabalho dos controladores na região do Controle de Aproximação de São Paulo (APP-SP) com o objetivo de destacar a importância de se adotar ações para a melhoria do tráfego aéreo na região. Nenhum desses trabalhos nacionais considerou os efeitos adversos das condições de tempo.

\subsection{Condições meteorológicas e tráfego aéreo}

Já há algum tempo a Federal Aviation Administration (FAA) e o EUROCONTROL Experimental Centre (EEC) vêm realizando estudos sobre a interferência das condições meteorológicas sobre o gerenciamento de tráfego aéreo e sobre a integração das previsões de tempo ao gerenciamento de fluxo. Essas organizações administram o National Airspace System (NAS) e o EUROCONTROL System, respectivamente. Esses estudos revelam como as condições atmosféricas podem impactar a operação das aeronaves em voo ou o funcionamento dos aeroportos ou órgãos ATC. Consequentemente, o fluxo de tráfego aéreo sofre com restrições e paralizações.

Para Kuln (2008), devem ser realizados esforços para desenvolver ferramentas de decisão-suporte relacionadas ao gerenciamento do tráfego aéreo. 0 aumento da segurança e eficiência do ATC deve ser o objetivo principal. Nesse contexto, as condições significativas de tempo não podem ser deixadas de lado. Nessa mesma linha, para Klein et al. (2009), as condições de tempo e a demanda por tráfego aéreo nunca devem ser consideradas separadamente, quando se analisa a ocorrência de impacto operacional nos aeroportos. Steiner et al. (2010) exploram uma nova abordagem para o uso de dados de modelo de previsão numérica de alta resolução no intuito de prever impactos probabilísticos para a aviação relacionados às condições de tempo.

Diversos trabalhos validam o quanto e como as condições de tempo impactam o sistema de transporte aéreo, no contexto da segurança, eficiência, previsibilidade, flexibilidade etc. Os trabalhos realizados por Krozel et al. (2011; 2007), Krozel (2011), Michalek e Balakrishnan (2010; 2009a; 2009b), Song et al. (2009), DeLaura et al. (2008), Kuln (2008), DeLaura e Evans (2006), Weber et al. (2007; 2005) 
e Rhoda et al. (2002), entre vários outros, focam na identificação e no emprego de técnicas para se evitar as condições convectivas de tempo durante o voo.

Outros estudos sobre a relação entre a condição de tempo e condições ATM podem ser encontrados em Klimenko e Krozel (2011) e Evans et al. (2005). Sobre a integração das condições meteorológicas em sistemas ATM, trabalhos como MIT (2016a; 2016b), Consiglio et al. (2011), Krozel (2011), FAA (2007) e Evans e Ducot $(2006,1994)$ são destaques.

\subsection{Simulação de tráfego aéreo}

Não é uma tarefa trivial identificar o nível do impacto provocado pelas condições meteorológicas sobre o fluxo de tráfego aéreo diário, considerando as diversas variáveis que envolvem este contexto. Nesse caso, destaca-se a possibilidade de ocorrência de diferentes tipos de condições meteorológicas adversas para a aviação, com diferentes intensidades, em horários distintos, proporcionando impactos variados. Assim, uma série de pesquisadores busca desenvolver ferramentas computacionais apropriadas na tentativa de realizar tal missão. Vários trabalhos validam tais ferramentas com o objetivo de representar o que acontece no decorrer de um dia de movimento do transporte aéreo.

EUROCONTROL (2016) apresenta algumas das principais ferramentas que tratam sobre simulação em tempo acelerado (Fast-time Simulation Tools). Nesse trabalho, o simulador TAAM é caracterizado como a principal delas, quando se estuda o desempenho das atividades de tráfego aéreo em área de controle terminal.

Majumdar e Polak (2014) propõem um método para determinar a capacidade do espaço aéreo da Europa, simulando a carga de trabalho do controlador de tráfego aéreo. Também Majumdar et al. (2005) simulam os impactos provocados por fatores relacionados ao tráfego aéreo sobre a carga de trabalho dos controladores e sobre os setores do espaço aéreo europeu, durante um dia completo, utilizando o Re-organized ATC Mathematical Simulator (RAMS).

EUROCONTROL (2000) realiza uma ampla avaliação sobre o modelo de simulação acelerada Total Airspace and Airport Modeller (TAAM), e EUROCONTROL (2003a) faz um estudo detalhado sobre os resultados produzidos por três modelos utilizados no EUROCONTROL CEATS. EUROCONTROL (2003b) descreve e destaca o uso da metodologia CAPAN (ATC Capacity Analyser tool) como uma poderosa metodologia para medidas precisas de carga de trabalho e capacidade, quando se usam simuladores e modelos matemáticos como ferramenta de trabalho.

Todas essas pesquisas reforçam o emprego sistematizado de simuladores na obtenção de resultados relacionados aos serviços ATC, determinando fatores intervenientes, inclusive a carga de trabalho dos controladores de tráfego aéreo.

\section{MATERIAIS E MÉTODOS}

Para obter resultados fidedignos, a realização de simulações de tráfego aéreo necessita de dados precisos, representativos do movimento aéreo desejado, e de um modelo confiável. Esses quesitos foram considerados nesta pesquisa e estão descritos a seguir.

\subsection{A base de dados de tráfego aéreo}

A base de dados sobre o tráfego aéreo foi obtida do arquivo de voos repetitivos e do arquivo do Horário de Transporte (HOTRAN), oriundos do CGNA e da ANAC, respectivamente. Eles se referem ao dia 10 de junho de 2014, uma terça-feira. Esse dia foi utilizado por ser o que melhor representa o número diário médio dos voos da semana, segundo procedimentos operacionais cotidianos estabelecidos pelo CGNA. Nesse contexto, foram considerados todos os voos de aeronaves comerciais de matrícula brasileira, em linhas domésticas e internacionais, e voos de empresas internacionais em voos regulares internacionais para a realização de todas as simulações deste trabalho. 


\subsection{A região de estudo}

A região de estudo escolhida e os setores de controle de tráfego aéreo da TMA-SP podem ser vistos na Figura 1. Os setores são definidos de acordo com a complexidade e/ou o fluxo do tráfego aéreo (SRPVSP, 2014). Os limites verticais dos setores utilizados nas simulações podem ser visualizados em Rocha (2017).

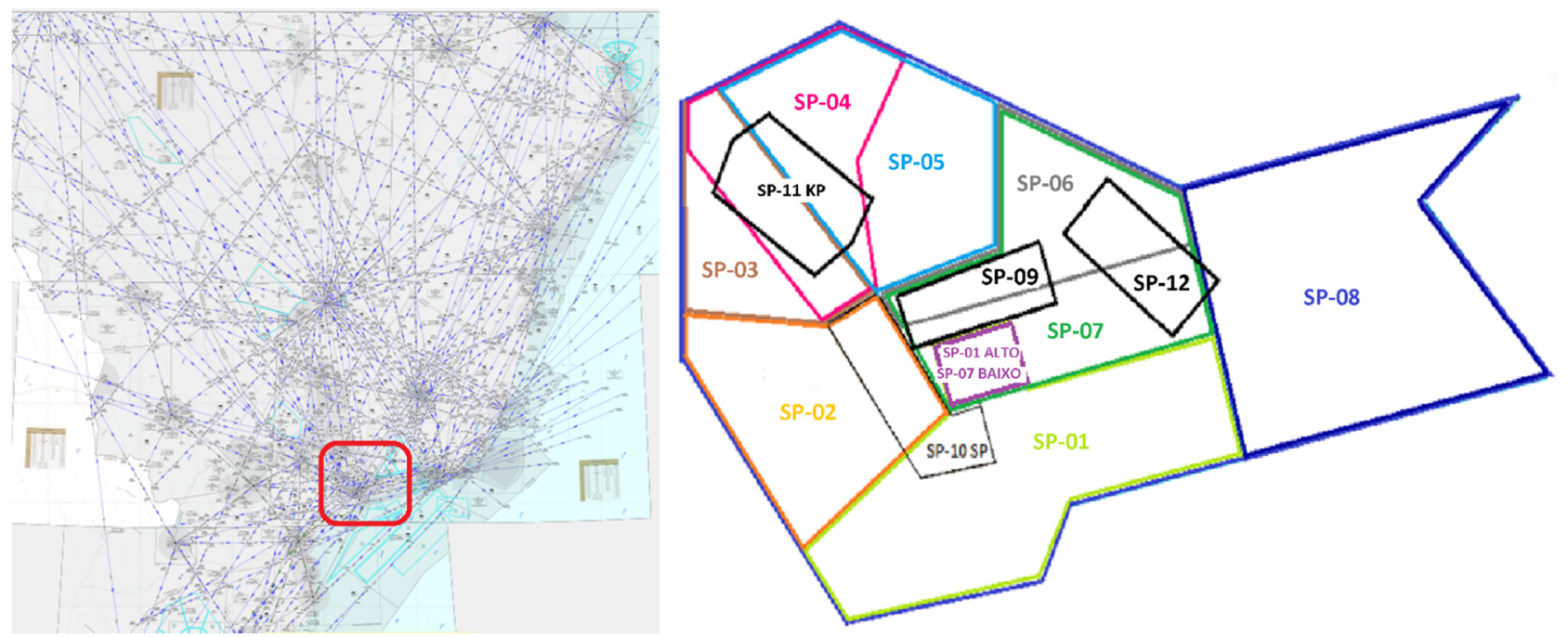

Figura 1. Detalhe de uma carta de rota, representando a área de controle terminal de São Paulo, com seus respectivos setores. Fonte: Plano de voo net. Disponível em: http://www.planodevoo.net/ index3.html e SRPV-SP, 2014.

\subsection{Relatórios diários de situação}

Informações básicas para a realização deste trabalho foram coletadas dos 365 relatórios diários de situação do ano de 2014, gerados pelo CGNA. São elas: procedimentos diários de gerenciamento de fluxo de tráfego aéreo relacionados às condições de mau tempo; atrasos médios dos voos maiores que 30 minutos, ocorridos nos aeroportos monitorados por esse centro; e as condições de mau tempo decorrentes das condições atmosféricas. Isso permitiu identificar quais os dias em que houve os maiores impactos, selecioná-los e realizar as rodadas de simulação, descritas na Tabela 1.

Tabela 1: Sete dias de maiores atrasos nas operações de pouso e decolagem nos aeroportos nacionais sob a coordenação do CGNA.

\begin{tabular}{cccccccc}
\hline \multicolumn{7}{c}{ Atrasos em Nível Nacional 2014 (maiores que 30 min.) } \\
\hline Rodada 1 - Referência & Rodada 2 & Rodada 3 & Rodada 4 & Rodada 5 & Rodada 6 & Rodada 7 & Rodada 8 \\
\hline 10 Jun & $24 \mathrm{Jan}$ & 25 Jan & $28 \mathrm{Fev}$ & $15 \mathrm{Abr}$ & 28 Jun & 17 Dez & 23 Dez \\
\hline--- & $22,26 \%$ & $23,23 \%$ & $23,88 \%$ & $26,80 \%$ & $23,30 \%$ & $23,80 \%$ & $28,18 \%$ \\
\hline
\end{tabular}

\subsection{Configuração e simulação}

As simulações foram realizadas utilizando-se a configuração-padrão (default), prevista pelo simulador TAAM. A versão desse software empregada foi a 3.0, release 12, 2015. Foram realizados os seguintes passos metodológicos com o objetivo de obter os resultados sobre a carga de trabalho dos controladores:

a) definição da área de estudo e de seus setores, ou seja, a TMA-SP, considerando a região do espaço aéreo entre o solo e 19.500 pés;

b) desenho da setorização do Espaço Aéreo Brasileiro contendo a TMA-SP;

c) criação do arquivo contendo aeroportos no Brasil;

d) criação de arquivo com todos os waypoints no espaço aéreo brasileiro;

e) criação das rotas, a fim de atender aos voos da amostra;

f) criação dos planos de voo, com base na lista de voos regulares nacionais e internacionais 
repetitivos;

g) criação de layouts dos principais aeroportos pertencentes à área de estudo (Guarulhos, São Paulo, Campinas);

h) criação das áreas de gerenciamento de fluxo em cada cenário;

i) criação dos procedimentos atualizados de voo para a saída e chegada nos aeroportos internacionais de São Paulo, Guarulhos e Campinas;

j) identificação dos sete principais dias onde o tráfego aéreo nacional sofreu os maiores atrasos;

k) identificação das condições meteorológicas utilizadas como referência no TAAM;

l) criação das áreas de desvio de mau tempo nos sete cenários considerados;

m) criação de regras de desvio para todos os cenários que possuíam condições meteorológicas adversas;

n) identificação e criação das ações de gerenciamento de fluxo específicas de mau tempo empregadas nos dias definidos para a simulação;

o) simulação e geração de relatórios de carga de trabalhos dos controladores para as oito rodadas, considerando o movimento de tráfego aéreo de uma terça-feira;

p) integração de arquivos para formar os oito cenários de simulação; e

q) seleção dos dados de interesse nos oito relatórios de simulação.

Mais detalhes sobre a metodologia de trabalho empregada podem ser encontrados em Rocha (2017).

\subsection{Condições meteorológicas}

Tomando como base os relatórios de situação produzidos pelo CGNA em 2014, foram selecionados os sete dias contidos na Tabela 1. As imagens de satélite presentes na Figura 2 apontam as condições meteorológicas das 18 UTC de cada um desses dias.

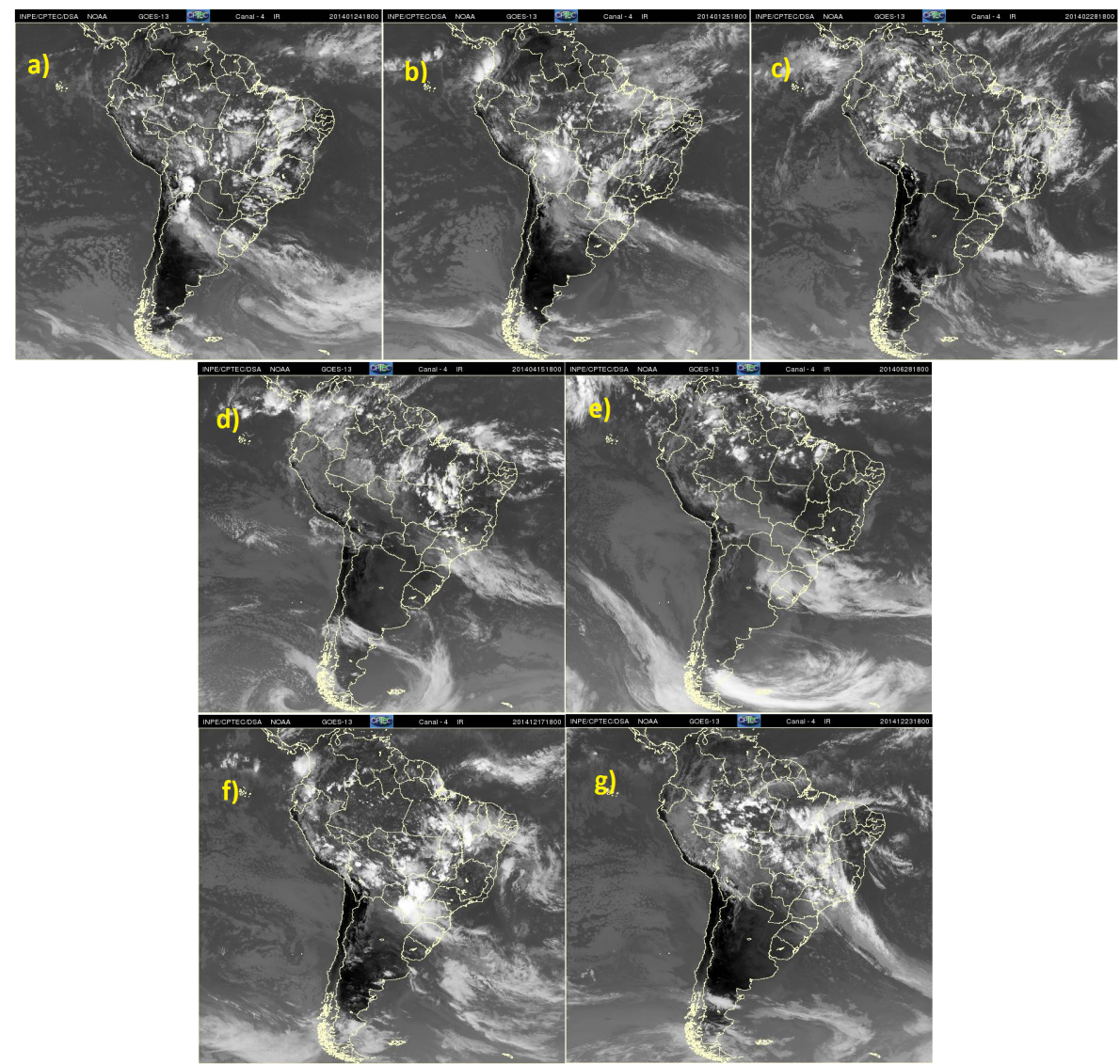

Figura 2. Imagens de satélites das 18 UTC. a) 24 de janeiro; b) 25 de janeiro; c) 28 de fevereiro; d) 15 de abril; e) 28 de junho; f) 17 de dezembro; g) 23 de dezembro. Fonte: CPTEC, (2016). 
Observando-se essa figura, é possível inferir que a região de estudo sofreu impacto direto das condições meteorológicas adversas em quatro dos sete dias selecionados. Nos outros três dias, diversos tipos de sistemas meteorológicos impactaram as condições de voo, provocando ações de gerenciamento de fluxo nas regiões de informação de voo de Curitiba, Brasília e Recife. Nesses casos, massas de ar frio e quente atuavam na região de estudo. Elas provocaram restrição de visibilidade e teto baixo pela manhã, em alguns aeroportos, e convecções intensas no período da tarde. A descrição detalhada de cada uma das condições sinóticas de tempo que justificam tais afirmativas pode ser encontrada em Rocha (2017).

\subsection{A metodologia de trabalho}

Cada uma das informações descritas no item 2.4 foi inserida no TAAM. Dessa forma, a partir de um momento predefinido, foi realizada uma primeira simulação do tráfego aéreo, sem levar em conta quaisquer condições meteorológicas. Ela foi definida como Rodada 1 - Referência. Em seguida, foram realizadas outras sete simulações, aqui descritas como Rodadas de 2 a 8, considerando-se os dias citados na Tabela 1 .

Nesses casos, apesar de os dias da semana possuírem números de voos diferentes entres si, utilizouse o movimento de tráfego aéreo semelhante ao de uma terça feira como base de trabalho, em função dessa condição melhor representar o número diário médio dos voos da semana. Isso foi feito para que os resultados encontrados pudessem ser comparados considerando-se apenas a variável relativa às condições de tempo adversas, desconsiderando a variável número de voos diários.

Além de suas próprias configurações, os procedimentos de gerenciamento de fluxo de tráfego aéreo exclusivos da presença das condições meteorológicas encontradas, bem como as regras, regulamentos, normas e procedimentos relacionados ao serviço e ao gerenciamento de tráfego aéreo estabelecidos pelo DECEA, fizeram parte da configuração do TAAM.

Os procedimentos relacionados aos desvios nos voos presentes nas regiões de mau tempo levaram em conta a existência de ecos de radar iguais ou superiores a $41 \mathrm{dBz}$, e topos com 25.000 pés ou superiores. Nesses casos, os desvios laterais realizados iniciaram com 25 NM dessa condição meteorológica (RHODA et al., 2002), contornando o mau tempo sempre pelo menor percurso. A intensidade dos ecos e os topos das nuvens convectivas foram definidos por meio das imagens do radar de São Roque - SP. Não foram realizados desvios para as aeronaves que voavam em níveis de voo iguais ou acima dos topos estabelecidos.

Não foi considerado o efeito do vento para a definição das cabeceiras das pistas, nem em qualquer outro nível da atmosfera, em todas as simulações realizadas. As cabeceiras das pistas utilizadas foram as mais usuais, considerando o vento predominante nos principais aeroportos envolvidos nas simulações.

Para a definição de carga de trabalho do controlador de tráfego aéreo, em minutos, este trabalho considerou a metodologia CAPAN, descrita em EUROCONTROL (2003b). Tendo em vista que o TAAM caracteriza a carga de trabalho por meio de parâmetro adimensional, este trabalho utilizou o que descreve EUROCONTROL (2000, p. 53) para a conversão desse parâmetro para a referência minutos. Nesse caso, um minuto de tempo de carga de trabalho encontrado pelo RAMS, e descrito na metodologia CAPAN, corresponde a dois pontos do valor encontrado pelo TAAM.

A existência de NOTAMs (Notice to Airmen), inoperâncias em auxílios à navegação, bem como quaisquer outras condições restritivas às operações aéreas não oriundas das condições de tempo adversas, também não foi considerada nas simulações.

A partir dessas considerações, o simulador TAAM utilizou sua própria dinâmica de gerenciamento de tráfego aéreo nas oito rodadas, determinando os resultados apresentados nos itens 3.1 e 3.2. 


\section{RESULTADOS E DISCUSSÃO}

\subsection{Estimativa da carga de trabalho nos setores da tma-sp, por rodada e por hora}

A Figura 3 apresenta a estimativa da carga de trabalho dos cenários diários utilizados nas oito rodadas de simulação nos setores da TMA-SP. As cores observadas na figura possuem os seguintes significados relativos à carga de trabalho: a) cinza - 0 a 10 minutos: carga de trabalho muito leve; b) azul claro - 11 a 17 minutos: carga de trabalho leve; c) amarelo - 18 a 31 minutos: carga de trabalho moderada; d) marrom - 31 a 41 minutos: carga de trabalho pesada; e) vermelho - igual ou superior a 42 minutos: sobrecarga de trabalho.

Considerando os resultados obtidos nas simulações, a metodologia CAPAN citada em EUROCONTROL (2003b, p. 5) e as condições sinóticas de tempo descritas em Rocha (2017) e nos Relatórios de Situação do CGNA, é possível observar que:

a) houve relativo aumento na estimativa da carga de trabalho dos controladores na grande maioria das rodadas e horários dos dias avaliados;

b) as células destacadas em vermelho apontam situações onde o limite de 42 minutos/hora foi atingido ou ultrapassado, fator este definido como sobrecarga de trabalho por EUROCONTROL (2003b, p. 5);

c) dois grupos de setores da Rodada 1 operaram em faixas de carga de trabalho distintas: os setores SP-06, SP-07, SP-08, SP-09 GR, e SP-10 SP com carga laboral próxima ao valor limite de 42 minutos/hora, e os demais setores com carga laboral inferior a 25 minutos;

d) as rodadas de simulação 2, 3, 5, 6 e 8 se destacam apresentando maiores quantidades de impactos de sobrecarga de trabalho por mais de uma hora consecutiva;

e) a Rodada 5 foi a mais impactada com sobrecarga de trabalho;

f) na Rodada 5, houve diminuição da carga de trabalho do setor SP-08 entre 09 UTC e 14 UTC, e aumento entre 16 UTC e 24 UTC. Também houve diminuição da carga de trabalho entre 07 UTC e 15 UTC e aumento entre 16 UTC e 21 UTC no setor SP-09 GR;

g) na Rodada 2, a alteração na carga de trabalho foi derivada de ações de gerenciamento de fluxo que tinham por objetivo manter o equilíbrio no número de voos presentes no setor SP-06, advindos das Regiões de Informações de Voo de Curitiba e Rio de Janeiro. Não foram percebidos impactos provocados por presença de condições de tempo significativas. A presença de mau tempo, a partir das 16 UTC, parece ter influenciado mais significativamente a carga laboral dos controladores de tráfego aéreo, principalmente nos setores SP-06 e SP-09 GR, somente entre 20 UTC e 21 UTC;

h) na Rodada 3, não foi possível perceber o grau de influência do mau tempo presente na TMA-SP sobre a elevação da carga de trabalho do controlador de tráfego aéreo ao se analisar os períodos do dia em questão. Nesse caso, foi possível observar condições de mau tempo em aeroportos e regiões do espaço aéreo fora da área de estudo. Percebe-se que parte dos atrasos identificados foi decorrente da existência de condições atmosféricas adversas ocorridas no dia 24 de janeiro, segundo o Relatório de Situação do CGNA desse dia;

i) na Rodada 5, onde ocorre o maior impacto das condições meteorológicas, havia a presença uma frente fria na região de estudo. Esse sistema promoveu condições que restringiram a visibilidade vertical e horizontal durante quase todo o dia nos principais aeroportos presentes na área estudada. Várias medidas de gerenciamento de fluxo foram adotadas para os voos que tinham destino ou saíam do aeroporto de Guarulhos, oriundos das ou para as Regiões de Informações de Voo de Curitiba, Brasília e Recife, além da Área de Controle Terminal do Rio de Janeiro (TMA-RJ). Nesse caso, percebe-se forte influência dessas condições adversas sobre a carga laboral dos controladores. Ela foi ultrapassada significativamente nos setores SP-08 e SP-09 GR, após as 16 UTC;

j) na Rodada 6, as condições atmosféricas apresentaram-se muito boas na área da TMA-SP, porém diversos aeroportos das regiões Sul, Sudeste, Centro-oeste e Nordeste sofreram com condições 
de teto baixo e restrição de visibilidade, devido à presença de sistemas meteorológicos que provocaram chuva e chuvisco nessas regiões. Alguns ficaram em condições abaixo dos mínimos operacionais em vários períodos do dia. Essa condição foi responsável por uma demanda reprimida e pelo consequente aumento do fluxo de aeronaves para a TMA-SP, principalmente nos horários destacados em vermelho; e

k) nas Rodadas 4 e 7 de simulação, observou-se a estimativa de aumento na carga laboral dos controladores de tráfego aéreo, porém não de intensidade suficiente para extrapolar a carga de trabalho, conforme definido em EUROCONTROL (2003b, p. 5) e DECEA (2014a, p. 15).

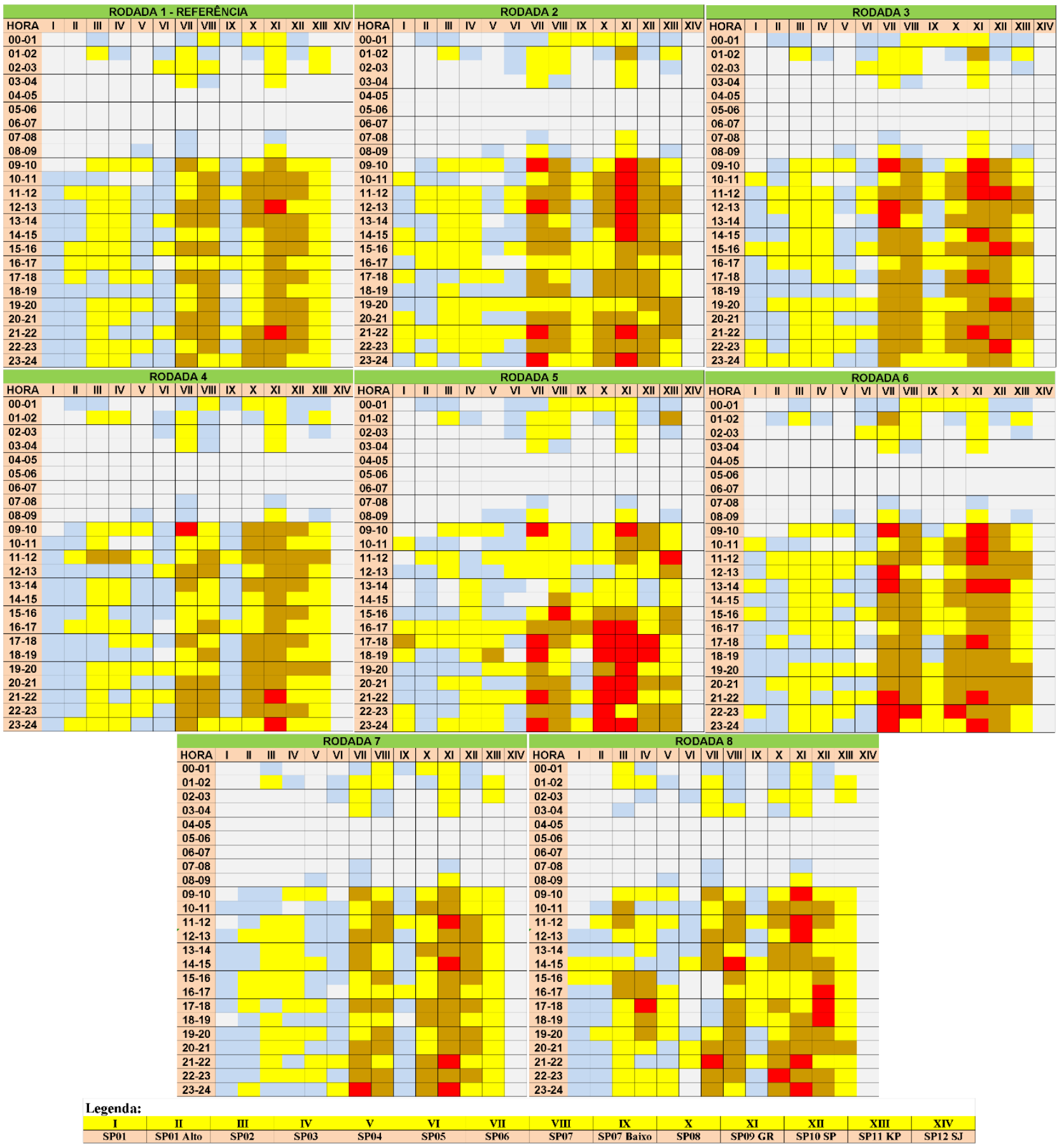

Figura 3. Estimativa da carga de trabalho dos cenários diários utilizados nas oito rodadas de simulação nos setores da TMA-SP, em minutos, considerando a presença de condições de tempo significativas. O eixo horizontal destaca a rodada considerada e os setores envolvidos. O eixo vertical indica os horários de ocorrência da carga de trabalho (em UTC). As células destacadas em vermelho representam cargas de trabalho iguais ou superiores ao valor limite de 42 minutos/hora, apresentado por EUROCONTROL (2003b, p. 5). 


\subsection{Estimativa da carga de trabalho na tma-sp, por setor e por hora}

Quando se analisa a Figura 4, utilizando-se o parâmetro relativo às cores já citados no item 3.1, é possível identificar alguns aspectos importantes relacionados à estimativa de carga de trabalho dos controladores de tráfego aéreo. São eles:

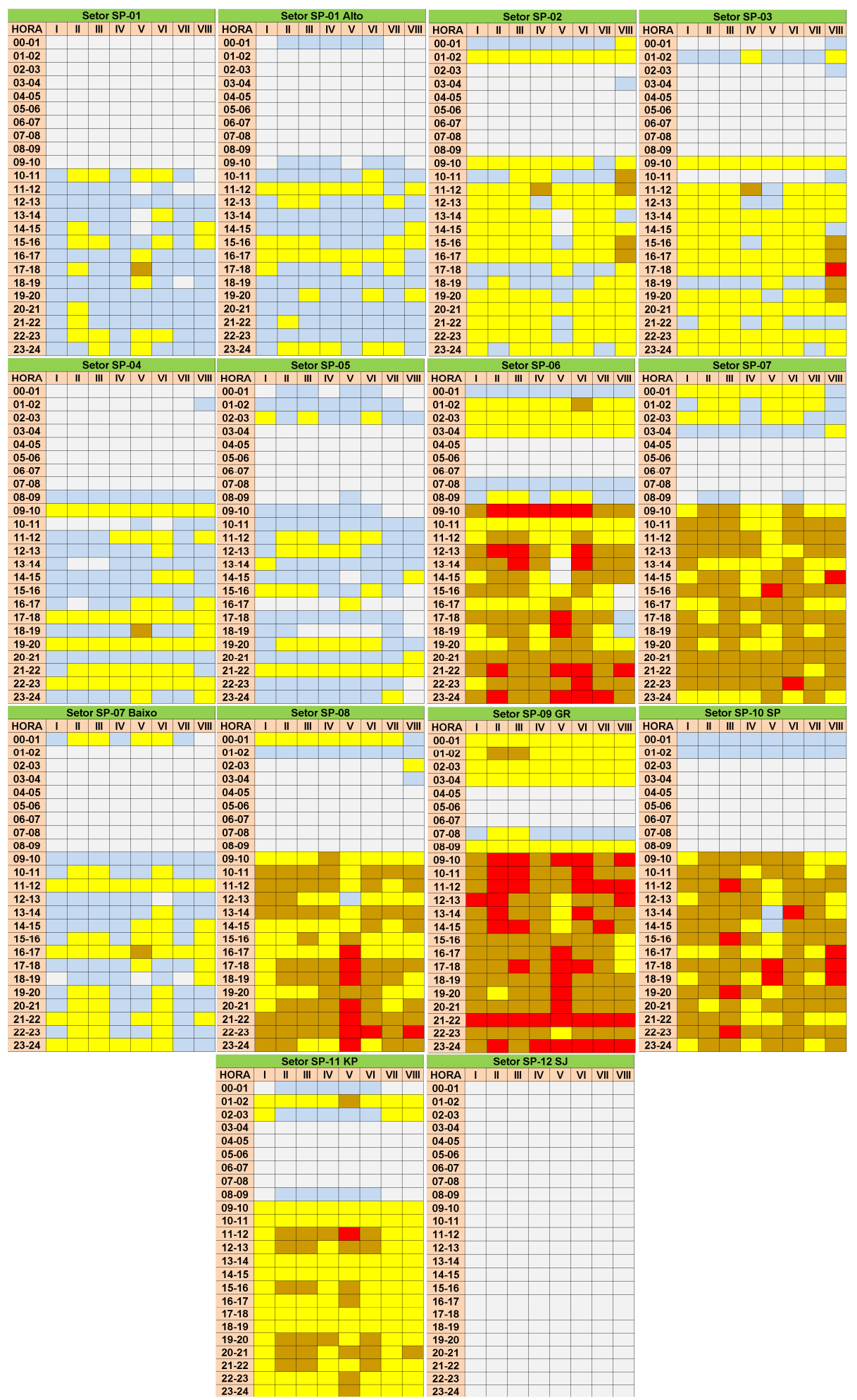

Figura 4. Estimativa de impacto na carga de trabalho do controlador de tráfego aéreo nos setores TMA-SP, em presença de condições de tempo significativo, em minutos. O eixo horizontal destaca a rodada considerada e os setores envolvidos. O eixo vertical indica os horários de ocorrência da carga de trabalho (em UTC). As células destacadas em vermelho representam cargas de trabalho superiores ao valor limite de 42 minutos/hora, sugeridos por EUROCONTROL (2003b, p. 5). 
a) as células destacadas em vermelho apontam várias situações onde o limite de 42 minutos/hora foi atingido ou ultrapassado, fator este definido como sobrecarga de trabalho por EUROCONTROL (2003b, p. 5);

b) considerando a Rodada 1 de referência, de modo geral, é possível perceber que:

- houve um aumento na carga laboral em quase todas as rodadas, setores e horários, excluindo-se desse quesito o setor SP-12 SJ;

- os setores SP-06, SP-07, SP-08, SP-09 GR, e SP-10 SP operaram na maioria dos horários com carga de trabalho acima de 25 minutos, a partir das 09 UTC;

c) os setores SP-06, SP-08, SP-09 GR e SP-10 SP são mais impactados em decorrência do aumento da carga laboral;

d) o setor SP-09 GR é o mais crítico, sendo o horário entre 21 UTC e 22 UTC o mais impactado;

e) alguns casos descritos no item "a" superam em pelo menos duas horas consecutivas o limite de 42 minutos/hora. Isso contraria aspectos operacionais descritos em DECEA (2014a, p. 15). São eles:

- o setor SP-06 com três momentos do dia: um na Rodada 3, entre 12 UTC e 14 UTC e dois na Rodada 6, entre 12 UTC e 14 UTC e entre 21 UTC e 24 UTC;

- o setor SP-08 com dois casos observados na Rodada 5, sendo um entre 16 UTC e 18 UTC e outro entre 20 UTC e 24 UTC;

- o setor SP-09 GR com cinco casos, distribuídos na Rodada 2, entre 10 UTC e 13 UTC; na Rodada 3, entre 09 UTC e 12 UTC; na Rodada 5, entre 16 UTC e 22 UTC; na Rodada 6, entre 09 UTC e 12 UTC; e na Rodada 8, entre 11 UTC e 13 UTC; e

- o setor SP-10 SP com dois casos distribuídos na Rodada 5, entre 17 UTC e 19 UTC; e na Rodada 8, entre 16 UTC e 19 UTC.

f) também podem ser observadas variações de diminuição de carga laboral. Nesse caso, as variações mais significativas estão nos setores SP-02 entre 11 UTC e 17 UTC na Rodada 5; SP-05 entre 15 UTC e 19 UTC na Rodada 8; SP-06 entre 13 UTC e 15 UTC na Rodada 5, e entre 15 UTC e 19 UTC na Rodada 8; e SP-10 SP entre 13 UTC e 15 UTC na Rodada 5.

\subsection{Discussão}

Diante dos resultados observados nas Figuras 3 e 4, é possível identificar alguns horários em que a carga de trabalho do ATCO atingiu ou superou o limite de 42 minutos/hora em duas ou mais horas consecutivas. Essas situações são consideradas inapropriadas por EUROCONTROL (2003b, p. 5). Esse fato é semelhante ao que é estabelecido por DECEA (2014a, p. 15) como sobrecarga de trabalho não controlada.

Assim, é possível perceber como a capacidade do espaço aéreo é impactada pelos movimentos das aeronaves em um determinado setor, em decorrência de condições adversas de tempo e ações de gerenciamento de fluxo, fato destacado principalmente nos trabalhos de Krozel et al. $(2011 ; 2007)$, Krozel (2011), Michalek e Balakrishnan (2010; 2009a; 2009b), Song et al. (2009), DeLaura et al. (2008), Kuln (2008), DeLaura e Evans (2006), Weber et al. (2007; 2005), e Rhoda et al. (2002), entre outros.

Através da definição do limite da carga de trabalho dos ATCOs, é possível identificar quais os setores da TMA-SP que estão em condições críticas de operação. Nesse caso, chamam a atenção os setores SP06, SP-07, SP-08, SP-09 GR, e SP-10 SP, sendo o SP-06 e o SP-09 GR os dois piores.

Em alguns setores e horários dos dias, também é possível perceber a diminuição da carga de trabalho dos ATCOs. Destaca-se a diminuição apresentada na letra "f" do item 3.2. Nesse contexto, observa-se o deslocamento da demanda de tráfego aéreo do horário da manhã, com maior presença de teto baixo e restrição de visibilidade, para a tarde, onde essas condições de tempo foram menos impactantes.

Como descrito anteriormente, as rodadas de simulação foram realizadas com informações de sete 
dias de condições adversas de tempo. Elas correspondem aos dias de maiores atrasos ocorridos no movimento do tráfego aéreo, em nível nacional. Observa-se que, dos sete casos selecionados, cinco ocorreram em meses de verão, o que concorda com o que dizem Robinson et al. (2004, citado por DeLaura et al., 2008). Para os outros dois, um ocorreu em um mês de outono e outro em um mês de inverno. Em todos esses casos, destaca-se a presença de condições adversas de tempo, dentro e/ou fora da área de estudo, que alteraram a complexidade dos setores controlados, o gerenciamento de fluxo das aeronaves em voo e, consequentemente, a carga de trabalho dos controladores.

Nesses termos, é possível observar que, dos sete dias selecionados, quatro possuíam sistemas sinóticos atuando dentro da área de interesse do estudo. Três deles sofriam atuação de massas de ar frio (inverno) ou quente (verão), produzindo efeitos adversos ao voo tal como restrições de teto e visibilidade pela manhã e convecções intensas à tarde. Nesses casos, quando a condição de mau tempo se localizava fora da região estudada, ela impactou aeroportos importantes e gerou demanda reprimida, os voos sofreram ações de gerenciamento de fluxo ao se aproximar e ao adentrar a TMA-SP, principalmente no período da tarde, em presença de nebulosidades convectivas. Condições de tempo e condições ATM foram amplamente estudadas por Klimenko e Krozel (2011) e Evans et al. (2005) e serviram de base para este trabalho.

No que diz respeito às condições de simulação, elas foram realizadas utilizando-se todos os procedimentos em vigor à época, definidos por DECEA (2014a; 2014b, 2014c; 2012; 2010), por SRPV-SP (2014) e por diversos procedimentos especiais contidos nas Circulares de Informações Aeronáuticas. Todas foram inseridas no TAAM, que é uma ferramenta de simulação acelerada considerada como o estado da arte para estudos sobre tráfego aéreo. Sua validação foi realizada por EUROCONTROL (2000).

Entretanto, algumas limitações devem ser consideradas nos resultados aqui obtidos. As rodadas de simulação não levaram em conta a presença do vento para a definição da operacionalidade das cabeceiras das pistas em uso, nem nos diversos níveis da atmosfera. Elas também não utilizaram como referência os procedimentos de desvios observados na operação real, e sim condições semelhantes ao que Rhoda et al. (2002) empregaram em seu trabalho.

Outro ponto importante a destacar é que o movimento de tráfego aéreo utilizado na simulação considerou somente os voos regulares de empresas nacionais e internacionais que voaram em 2014. Voos da aviação geral e militar não fizeram parte deste estudo.

NOTAMs e inoperâncias em auxílios à navegação, bem como quaisquer outras condições restritivas às operações aéreas, também não foram consideradas nas simulações.

Por fim, o movimento do tráfego aéreo utilizado nas rodadas de simulação considerou um dia específico da semana, ou seja, uma terça feira, sendo que esse movimento varia diariamente de acordo com o que pode ser observado, principalmente, nos HOTRANs do ano de 2014. A decisão de se adotar essa abordagem no trabalho foi tomada, principalmente, em função da necessidade de os resultados encontrados poderem ser melhor comparados entre si, considerando-se apenas uma variável: as condições de tempo adversas. Além disso, existe grande complexidade em se realizar as simulações quando se leva em conta todos os aspectos peculiares de cada dia, descritos no item 2.4. 0 tempo disponível para o trabalho também foi fator crítico.

Dessa forma, os resultados obtidos merecem atenção por parte dos planejadores do espaço aéreo nacional, dos gerentes de fluxo que atuam no SISCEAB e dos supervisores de salas operacionais presentes no APP-SP e Centros de Controle de Área (Area Control Center - ACC).

Eles demonstram a necessidade de se realizar mais estudos sobre esse assunto e apontam para a necessidade de se reavaliar a estrutura atual dos setores existentes na TMA-SP. Entende-se ainda ser necessário buscar ferramentas operacionais que agreguem valor às informações meteorológicas utilizadas para a tomada de decisão no gerenciamento de fluxo de tráfego aéreo nacional. Nesse contexto, destaca-se a necessidade de se desenvolver pesquisas para produzir sistemas que considerem a presença de condições adversas de tempo, tal como pode ser visto em MIT (2016a, 2016b), Consiglio et al. (2011), FAA (2007) e Evans e Ducot $(2006,1994)$. 


\section{CONCLUSÃO}

Este trabalho estimou como algumas condições atmosféricas adversas ao voo podem afetar a carga de trabalho do controlador de tráfego aéreo na TMA-SP. Isso foi feito pela utilização da ferramenta de simulação TAAM, definida com configurações-padrão do sistema (default). Nela foram inseridas informações relativas às regras de tráfego aéreo vigentes em 2014 e às condições de mau tempo em dias, horários e setores específicos da TMA-SP. Nas rodadas de simulação, foram consideradas ainda todas as ações de gerenciamento de fluxo empregadas pelo CGNA, a nível nacional, exclusivamente derivadas da presença dessas condições meteorológicas significativas.

De modo geral, os resultados apontam para um aumento na carga de trabalho do controlador na maioria das rodadas e setores estudados. Entretanto, deve-se destacar que os maiores e principais impactos negativos ocorreram nos setores SP-06 e SP-09-GR, quando se leva em conta o limite definido por EUROCONTROL (2003b, p. 5) como sobrecarga de trabalho. Isso ocorreu em vários horários onde as condições adversas de tempo estiveram presentes, mesmo após a aplicação de medidas de gerenciamento de fluxo, fato que pode ser considerado como fator contribuinte forte na produção dos maiores atrasos detectados no movimento aéreo dos dias estudados. 0 principal horário que merece atenção está entre 21 UTC e 22 UTC no setor SP-09 GR.

Nesse contexto, é possível inferir que esses resultados podem auxiliar no estabelecimento de estratégias de emprego para pessoal ATCO por parte dos gestores operacionais das FMC impactadas, bem como do APP-SP. Nesse caso, os gestores poderão levar em conta as demandas horárias decorrentes de condições meteorológicas adversas nos setores identificados nesta pesquisa, quando houver casos semelhantes previstos ou observados. Além disso, as informações aqui obtidas poderão ser utilizadas durante uma possível reanálise da estrutura vertical e horizontal e da organização dos setores hoje estabelecidos, com o propósito de melhorar ainda mais a condição das rotas de chegadas e/ou partidas para aeroportos presentes na TMA-SP.

No contexto acadêmico, este estudo aponta para a necessidade de se realizar pesquisas que envolvam o simulador TAAM e os diversos tipos de condições meteorológicas adversas em rota, em TMAs ou em aeroportos, com o objetivo de estabelecer regras padronizadas que solucionem possíveis gargalos operacionais. Com a integração dessas regras ao sistema ATM, haverá redução da subjetividade e dos efeitos indesejáveis produzidos por decisões operacionais ineficientes quando em presença de diversos tipos de mau tempo para a aviação, além de diminuir o número de medidas ATM subjetivas por parte do CGNA, das FMC e dos controladores nas posições operacionais, diminuindo suas respectivas cargas de trabalho.

É importante dizer ainda que, neste estudo, o fluxo de tráfego aéreo simulado considerou apenas o movimento de aeronaves previsto nos planos de voo repetitivos, ou seja, somente o tráfego aéreo civil regular. Isso sugere que os resultados poderão ser significativamente majorados quando da realização de simulações que considerem também os demais tipos de aviação (voos não regulares e aviação militar).

Algumas limitações neste trabalho devem ser consideradas:

- durante as simulações, não se levou em conta a presença do vento para a definição da operacionalidade das cabeceiras das pistas em uso nem nos diversos níveis da atmosfera;

- os desvios realizados nas rodadas de simulação não consideraram a condição real praticada operacionalmente e sim os procedimentos observados por Rhoda et al. (2002);

- NOTAMs e inoperâncias em auxílios à navegação, bem como quaisquer outras condições restritivas às operações aéreas, também não foram consideradas nas simulações; e

- o movimento do tráfego aéreo utilizado toma como referência um dia específico da semana, sendo que ele varia diariamente de acordo com o que pode ser observado nos HOTRANs do ano de 2014. Nesse caso, diferentes ações de gerenciamento de fluxo de tráfego aéreo podem ser empregadas de acordo com a intensidade do tráfego, principalmente em presença de condições de tempo adversas. Isso não foi levado em conta no trabalho. 
Assim, apesar de os resultados obtidos ainda carecerem de mais estudos, eles são de grande relevância para a adoção de ações táticas operacionais no CGNA, bem como para abrir novas linhas de pesquisas acadêmicas que relacionem condições meteorológicas às atividades de gerenciamento de tráfego aéreo. Isso acabará por contribuir com novas propostas de planejamento da segurança e eficiência operacional, no contexto do transporte aéreo nacional.

Além disso, é importante que os órgãos responsáveis pelo tráfego aéreo iniciem ações que possam desenvolver um sistema integrado que considere as condições meteorológicas e as ações táticas adotadas no contexto do gerenciamento de fluxo do tráfego aéreo nacional. Isso poderá minimizar sobremaneira os impactos provocados sobre os atrasos e, consequentemente, sobre as empresas aéreas, bem como sobre os usuários desse meio de transporte de significativa importância para a economia do Brasil.

\section{REFERÊNCIAS}

ANAC (2014). Anuário do Transporte aéreo 2014. Brasília, ANAC. Disponível em: <http://www.anac.gov.br/assuntos/dados-eestatisticas/mercado-de-transporte-aereo/anuario-do-transporte-aereo/dados-do-anuario-do-transporte-aereo>. Acesso em: 19/12/2017.

BARNHART, C.; D. FEARING; A. ODONI e V. VAZE (2012) Demand and capacity management in air transportation. Research Paper. EURO J Transp Logist, v. 1, questões 1-2, p.135-155. DOI 10.1007/s13676-012-0006-9. Disponível em: $<$ https://link.springer.com/content/pdf/10.1007\%2Fs13676-012-0006-9.pdf>. Acesso em: 19/12/2017.

BAUM, D. M. e C. MÜLLER (2008) Análise da relação a carga de trabalho do APP-SP com a complexidade da TMA-SP, através de ferramenta computacional. Sitraer 7, Ano 2008, Tr. 436, p. 469-481. Disponível em: <https://cabecadepapel.com/sites/viisitraer2008/pdf/436.pdf>. Acesso em: 19/12/2017.

CGNA (2014) Relatórios de Situação. Departamento de Controle do Espaço Aéreo. Rio de Janeiro: DECEA.

CPTEC (2016) Imagens de Satélites. Acervo de Imagens. Instituto Nacional de Pesquisas Espaciais. Cachoeira Paulista: INPE. Disponível em: <http://satelite.cptec.inpe.br/acervo/goes.formulario.logic>. Acesso em: 27/03/2016.

CONSIGLIO, M. C.; J. P. CHAMBERLAIN e S. R. WILSON (2011) Integration of Weather Avoidance and Traffic Separation. 30th Digital Avionics Systems Conference (DASC). IEEE/AIAA. DOI: 10.1109/DASC.2011.6096050. Disponível em: <https://ntrs.nasa.gov/archive/nasa/casi.ntrs.nasa.gov/20110020261.pdf>. Acesso em: 19/12/2017.

DELAURA, R.; M. ROBINSON; M. PAWLAK; J. E. EVANS (2008) Modeling Convective Weather Avoidance in Enroute Airspace. 13th Conference on Aviation, Range, and Aerospace Meteorology (ARAM), New Orleans, Louisiana. Disponível em: $<$ https://ams.confex.com/ams/pdfpapers/132903.pdf>. Acesso em: 19/12/2017.

DELAURA, R. e J. E. EVANS (2006) An exploratory study of modeling enroute pilot convective storm flight deviation behaviour, 12th Conference on Aviation, Range, and Aerospace Meteorology (ARAM), Atlanta, Georgia. Disponível em: <https://www.ll.mit.edu/mission/aviation/publications/publication-files/nasa-reports/DeLaura_2006_NASAA6_WW-12670.pdf>. Acesso em: 19/12/2017.

DECEA (2014a) Capacidade do espaço aéreo. ICA 100-17. Rio de Janeiro: DECEA. Publicado no Boletim do Comando da Aeronáutica no 160, de 16 de agosto de 2014. Disponivel em: <https://publicacoes.decea.gov.br/>. Acesso em: 19/12/2017.

DECEA (2014b) Regras do ar. ICA 100-12. Rio de Janeiro: DECEA. Publicado no Boletim do Comando da Aeronáutica no 160, de 26 de agosto de 2014. Disponivel em: <https://publicacoes.decea.gov.br/>. Acesso em: 19/12/2017.

DECEA (2014c) Serviços de tráfego aéreo. ICA 100-37. Rio de Janeiro: DECEA. Publicado no Boletim do Comando da Aeronáutica no 92, de 19 de maio de 2014. Disponivel em: <https://publicacoes.decea.gov.br/>. Acesso em: 19/12/2017.

DECEA (2012) Plano de Voo. ICA 100-11. Rio de Janeiro: DECEA. Publicado no Boletim do Comando da Aeronáutica nº 210, de 05 de novembro de 2012. Disponivel em: <https://publicacoes.decea.gov.br/>. Acesso em: 19/12/2017.

DECEA (2010) Serviço de gerenciamento de fluxo de tráfego aéreo. ICA 100-22. Rio de Janeiro: DECEA. Publicado no Boletim do Comando da Aeronáutica no 108, de 11 de junho de 2010. Disponivel em: <https://publicacoes.decea.gov.br/>. Acesso em: $19 / 12 / 2017$.

ERZBERGER, H. (2004) Transforming the NAS - The Next Generation Air Traffic Control System. Ames Research Center, Moffett Field, California. NASA/TP-2004-212828. Disponível em: <https://www.aviationsystemsdivision.arc.nasa.gov/publications/tactical/NASA-TP-2004-212828.pdf>. Acesso em: $19 / 12 / 2017$.

EUROCONTROL (2016) Fast-time Simulation Tools. Disponível em: <http://www.eurocontrol.int/eec/public/standard_page/ WP_Fast_Time_Simulation_Tools.html >. Acesso em: 10/02/2016.

EUROCONTROL (2003a) Comparison of Different Workload and Capacity Measurement Methods Used in CEATS Simulations: Comparison of SAAM 3; FTS 3, SSRTS3 and CEATS2007 UAC Capacity. Research, Development and Simulation Centre. Budapest - Hungary. EUROCONTROL. Disponível em: <http://www1.atmb.net.cn/CD_web/UploadFile/2013092718150954.pdf>. Acesso em: 19/12/2017.

EURoConTROL (2003b) Pessimistic Sector Capacity Estimation. EEC Note № 21/03. Project COCA. Network Capacity and Demand Management - NCD. Centre de Bois des Bodes. France. Disponível em: <https://www.eurocontrol.int/eec/public/standard_page/DOC_Report_2003_026.html>. Acesso em: 19/12/2017. 
EUROCONTROL (2000) TAAM Operational Evaluation. EEC Report N 351. Project SIM-S-E8. Aug. 2000.

EVANS, J. E. e E. R. DUCOT (2000) Corridor integrated weather system. MIT Lincoln Laboratory Journal, v. 16, n. 1, p. 59-80. Disponível em: <https://www.ll.mit.edu/publications/journal/pdf/vol16_no1/16_1_4EvansDucot.pdf>. Acesso em: 19/12/2017.

EUROCONTROL (1994) The Integrated Terminal Weather System. MIT Lincoln Laboratory Journal, v. 7, n. 2. Disponível em: <https://www.ll.mit.edu/mission/aviation/faawxsystems/itws.html>. Acesso em: 19/12/2017.

EVANS, J. E.; M. ROBINSON e S. ALLAN (2005). Quantifying convective delay reduction benefits for weather/ATM systems. 6th EUROCONTROL/FAA ATM R\&D Seminar, v. 14, n, 1, p. 69-93. Baltimore. Disponpivel em: $<$ https://arc.aiaa.org/doi/abs/10.2514/atcq.14.1.69?journalCode=atcq>. Acesso em: 19/12/2017.

FAA (2007) Report of the Weather-ATM Integration Working Group. Research, Engineering and Development Advisory Committee. Oct, 2007. Disponível em:

<https://www.faa.gov/about/office_org/headquarters_offices/ang/offices/tc/about/campus/faa_host/RDM/media/ pdf/Report-WeatherWorkingGroup.pdf >. Acesso em: 03/02/2016.

KLEIN, A.; S. KAVOUSSI, e R. S. LEE (2009) Weather forecast accuracy: study of impact on airport capacity and estimation of avoidable costs. 8th USA/Europe Air Traffic Management Research and Development Seminar (ATM2009), Napa, California. Disponível em: <https://pdfs.semanticscholar.org/225b/702c488fcb697b16f4bbcbf78c23787c2680.pdf>. Acesso em: 19/12/2017.

KLIMENKO, V. e J. KROZEL (2011) Clear-Air Turbulence Impact Modeling Based on Flight Route Analysis, AIAA Guidance, Navigation, and Control Conference, Guidance, Navigation, and Control. Chicago, IL. DOI: 10.2514/6.2011-6513. Disponível em: <https://arc.aiaa.org/doi/abs/10.2514/6.2011-6513>. Acesso em: 19/12/2017.

KROZEL, J. (2011) Summary of Weather-ATM Integration Technology. Second Aviation, Range and Aerospace Meteorology Special Symposium on Weather-Air Traffic Management Integration, American Meteorological Society, Seattle, WA. Disponível em: <https://ams.confex.com/ams/91Annual/webprogram/Paper187588.html>. Acesso em: 19/12/2017.

KROZEL, J.; M. GANJI; S. YANG; J. S. B. MITCHELL e V. POLISHCHUK (2011) Metrics for Evaluating The Impact Of Weather On Jet Routes. 15th Conference on Aviation, Range, and Aerospace Meteorology. 9 p. Disponível em: <http://ams.confex.com/ams/14Meso15ARAM/webprogram/Manuscript/Paper191127/AMS11\%20ARAM\%20Rout e\%20Metrics\%20FINAL\%20081611.pdf>. Acesso em: 19/12/2017.

KROZEL, J.; J. S. B. MITCHELL; V. POLISHCHUK e J. PRETE (2007) Maximum Flow Rates for Capacity Estimation in Level Flight with Convective Weather Constraints. Air Traffic Control Quarterly, v. 15, n. 3, p. 209-238. DOI: 10.2514/atcq.15.3.209. Disponível em: <https://arc.aiaa.org/doi/abs/10.2514/atcq.15.3.209>. Acesso em: $19 / 12 / 2017$.

KULN, K. (2008) Analysis of thunderstorm effects on aggregated aircraft trajectories. Journal of aerospace computing, information, and communication. Aerospace Engineer, v. 5, n. 4, p.108-119, NASA Ames Research Center, Moffett, CA 94035. April, 2008. Disponível em: <https://arc.aiaa.org/doi/abs/10.2514/1.34830>. Acesso em: 19/12/2017.

MAJUMDAR, A. e J. W. POLAK (2014) Estimating Capacity of Europe's Airspace Using a Simulation Model of Air Traffic Controller Workload. Transportation Research Record Journal of the Transportation Research Board. Vol.1744. Imperial College London, Londinium, England, United Kingdom. DOI: 10.3141/1744-05. 2014. Disponível em: <http://trrjournalonline.trb.org/doi/abs/10.3141/1744-05>. Acesso em: 19/12/2017.

MAJUMDAR, A.; OCHIENG, W. Y.; MCAULEY, G.; LENZI, J. M.; LEPADATU, C. (2005) The Factors Affecting Airspace Capacity in Europe: A Framework Methodology Based on Cross Sectional Time-Series Analysis Using Simulated Controller Workload Data. 6th Seminar, v. 57, n. 3, paper 119, p. 385-405, Baltimore, MD, USA. DOI: 10.1017/S0373463304002863. Disponivel em: <http://www.atmseminar.org/seminarContent/seminar6/papers/p_119_U.pdf>. Acesso em: 19/12/2017.

MIT (2016a) FAA Weather Systems. Lincoln Laboratory. Disponível em: $<$ https://ll.mit.edu/mission/aviation/faawxsystems/faawxsystems.html>. Acesso em: 21/09/2017.

MIT (2016b) Weather - Air Traffic Management Integration. Lincoln Laboratory. Disponível em: <https://ll.mit.edu/mission/aviation/wxatmintegration/wxatminteg.html >. Acesso em: 21/09/2017.

MICHALEK, D. e H. BALAKRISHNAN (2010) Dynamic Reconfiguration of Terminal Airspace During Convective Weather. 49th IEEE Conference on Decision and Control (CDC). Atlanta, GA, USA. p. 4875-4881. DOI: 10.1109/CDC.2010.5718007. Disponível em: <http://ieeexplore.ieee.org/document/5718007/>. Acesso em: 19/12/2017.

MICHALEK, D. e H. BALAKRISHNAN (2009a) Building a Stochastic Terminal Airspace Capacity Forecast from Convective Weather Forecasts. In Proceedings of the Aviation, Range and Aerospace Meteorology Special Symposium on WeatherAir Traffic Management Integration. Disponível em: <http://web.mit.edu/hamsa/www/pubs/MichalekBalakrishnanAMS09.pdf>. Acesso em: 19/12/2017.

MICHALEK, D. e H. BALAKRISHNAN (2009b) Identification of Robust Routes using Convective Weather Forecasts. Eighth USA/Europe Air Traffic Management Research and Development Seminar, v. 46, n. 1, p. 56-73, Napa, California. Paper 124. DOI:10.1287/trsc.1110.0372. Disponível em: <http://www.mit.edu/ hamsa/pubs/MichalekBalakrishnanATM09.pdf>. Acesso em: 19/12/2017.

MOGFORT, R. H.; J. A. GUTTMAN; S. L. MORROW e P. KOPARDEKAR (1995) The Complexity Construct in Air Traffic Control: A Review and Synthesis of the Literature. U.S. Department of Transportation. Federal Aviation Administration. Office of Aviation Research. Washington, D.C. 20591. Disponível em: <http://www.tc.faa.gov/acb300/techreports/tn9522.pdf>. Acesso em: 19/12/2017.

PLANO DE VOO NET (2016) Carta ENRC Low. Disponível em: <http://www.planodevoo.net/index3.html>. Acesso em: $11 / 02 / 2016$. 
RHODA, D. A.; E. A. KOCAB e M. L. PAWLAK (2002) Aircraft encounters with convective weather in en route vs. terminal airspace above Memphis, Tennessee, 10th Conference on Aviation, Range and Aerospace Meteorology, American Meteorological Society, Portland, OR. Disponível em: <https://www.ll.mit.edu/mission/aviation/publications/publication-files/mspapers/Rhoda_2002_ARAM_MS-15308_WW-16138.pdf>. Acesso em: 19/12/2017.

ROCHA, A. C. (2017) Impacto Provocado pelas Condições Meteorológicas Sobre a Carga de Trabalho dos Controladores de Tráfego Aéreo e Sobre as Emissões de CO2 nas Áreas Terminais de São Paulo e Rio de Janeiro. versão: 2017-10-11. 244 p. IBI: <8JMKD3MGP3W34P/3NNAGG5>. Tese (Doutorado em Meteorologia) - Instituto Nacional de Pesquisas Espaciais (INPE), São José dos Campos. Disponível em: <http://urlib.net/8JMKD3MGP3W34P/3NNAGG5>. Acesso em: $17 / 10 / 2017$.

SRPV-SP (2014) Modelo operacional do controle da aproximação de São Paulo (APP-SP). São Paulo: SRPV-SP. Portaria SRPV no 102/OTNO, de 24 de abril de 2014.

SONG, L.; D. GREENBAUM e C. WANKE (2009) The Impact of Severe Weather on Sector Capacity. 8th USA/Europe Air Traffic Management Research and Development Seminar, Napa, CA. Disponível em: <http://www.atmseminarus.org/seminarContent/seminar8/papers/p_075_W.pdf>. Acesso em: 19/12/2017.

STEINER, M.; D. MEGENHARDT; R. BATEMAN e J. KROZEL (2010) Translation of Ensemble Weather Forecasts into Probabilistic Air Traffic Capacity Impact. Air Traffic Control Quarterly, v. 18, n. 3, p. 229-254. DOI: 10.2514/atcq.18.3.229. Disponível em: <http://ieeexplore.ieee.org/document/5347538/>. Acesso em: 19/12/2017.

TEIXEIRA, R. J. G. (2007) Análise do impacto do uso da re-setorização dinâmica na carga de trabalho do controlador de tráfego aéreo por meio de simulações computacionais. Dissertação (Mestrado). Escola Politécnica da Universidade de São Paulo. Departamento de Engenharia da Computação e Sistemas Digitais. São Paulo. Disponível em: <http://www.teses.usp.br/teses/disponiveis/3/3141/tde-09012008-162108/en.php>. Acesso em: 19/12/2017.

TOBARUELA, G.; A. MAJUMDAR e W. Y. OCHIENG (2012) Identifying Airspace Capacity Factors in the Air Traffic Management System. ATACCS'2012. DOCTORAL CONSORTIUM. London, UK. Disponível em: <http://www.halasesar.net/sites/default/files/documents/p219-tobaruela.pdf>. Acesso em: 19/12/2017.

WEBER, M. E.; J. E. EVANS; W. R. MOSER e O. J. NEWELL (2007) Air Traffic Management Decision Support During Convective Weather, MIT Lincoln Laboratory Journal, v. 16, n. 2. Disponível em: <https://www.ll.mit.edu/publications/journal/pdf/vol16_no2/16_2_03Weber.pdf>. Acesso em: 19/12/2017.

WEBER, M. E.; J. E. EVANS; M. WOLFSON; R. DELAURA; B. MOSER; B. MARTIN; J. WELCH; J. ANDREWS e D. BERTSIMAS (2005) Improving Air Traffic Management During Thunderstorms, 24th AIAA/IEEE Digital Avionics Systems Conference (DASC), Vol 1. Washington, DC. DOI: 10.1109/DASC.2005.1563353. Disponível em: https://www.researchgate.net/publication/4206227_Improving_air_traffic_management_during_thunderstorms. Acesso em: 19/12/2017. 